Pacific

Journal of

Mathematics

AREAS OF LEVEL SETS OF DISTANCE FUNCTIONS INDUCED BY ASYMMETRIC NORMS

DAVID G. CARABALLO 


\title{
AREAS OF LEVEL SETS OF DISTANCE FUNCTIONS INDUCED BY ASYMMETRIC NORMS
}

\author{
DAVID G. CARABALLO
}

\begin{abstract}
We establish useful upper bounds for the $(n-1)$-area of a level set $\rho^{-1}\{r\}$ of a general distance function $\rho$ to an $(n-1)$-dimensional compact subset $C$ of $\mathbb{R}^{n}$, in terms of $r$ and the area of $C$. These bounds nicely complement general isoperimetric inequalities that provide lower bounds for the same area. We allow distance functions induced from asymmetric norms, and prove our results without assuming that $C$ is smooth. Unlike standard upper bounds using Federer's Coarea Formula, which hold only for some values of $\boldsymbol{r}$ and which become arbitrarily large if we restrict $\boldsymbol{r}$ to be contained in sufficiently small intervals, our estimates hold for $\mathscr{L}^{1}$-almost every $\boldsymbol{r}>0$.

Our main result both extends and improves upon an important result of Almgren, Taylor, and Wang. First, our estimates hold for general distance functions. Second, in the case of ordinary distance functions, our estimates are sharper than theirs. Because our estimates hold for $\mathscr{L}^{\mathbf{1}}$-almost every $r$, we can easily integrate to obtain volume estimates, such as those typically required for Hölder continuity theorems for flows in $\mathbb{R}^{n}$. Indeed, Almgren, Taylor, and Wang used a weaker inequality to establish their main Hölder continuity theorem for curvature-driven flow of the boundary of a single crystal. In that setting, our estimate would lead to a similar result, but with a better coefficient.

We also establish several general results about asymmetric norms and their associated distance functions to compact sets. For example, the latter are Lipschitz continuous and have, for $\mathscr{L}^{n}$-almost every $x \in \mathbb{R}^{n}$, gradients with norms bounded $a$ priori from above and below.
\end{abstract}

\section{Introduction}

Suppose $C$ is any nonempty compact subset of $\mathbb{R}^{n}$, such as the boundary of an $n$-dimensional compact subset $K \subset \mathbb{R}^{n}$, or just a piece of a surface. It might just consist of a few points.

Suppose $\rho=\operatorname{dist}(\cdot, C)$ is the usual Euclidean distance function to the set $C$, and let

$$
\rho^{-1}\{r\}=\left\{x \in \mathbb{R}^{n}: \rho(x)=r\right\}
$$

denote the $r$-level sets of $\rho$.

MSC2000: primary 28A75, 52A40; secondary 46B20, 49Q10, 49Q15. 
If we let $r=r(t) \geq 0$ (with $r(0)=0$ ), we can define a flow in $\mathbb{R}^{n} \times \mathbb{R}_{+}$by setting

$$
C(t)=\rho^{-1}\{r(t)\} \text { for } t \geq 0 .
$$

If $C=\partial K$, we could use this formulation to model a set $K \in \mathbb{R}^{n}$ which is shrinking or expanding uniformly. If $r(t)$ is a nondecreasing function, we can model a front expanding uniformly away from $C$ in general. For such isotropic growth, this model could apply.

In practice, the conditions favorable for motion of a front may be quite different in different directions. For example, we may have a front moving across a medium with variable bulk density or concentration, or we might have a crystal lattice that prefers certain orientations.

In order to formally incorporate some anisotropy, we use general distance functions associated with asymmetric norms.

In Section 2 we introduce asymmetric norms and give some of their key properties (Theorem 2). We discuss general distance functions (defined using asymmetric norms) in Section 3. We show that such functions are within a constant factor of Euclidean distance (Theorem 3), are Lipschitz continuous (Theorem 4), and have gradients $\mathscr{L}^{n}$-almost everywhere having norms bounded a priori from above and below (Theorem 5).

We are thus led to studying nonuniform flows $C(t)=\rho^{-1}\{r(t)\}$ associated with $\Psi$-induced distance functions $\rho$ of the form

$$
\rho(x)=\inf _{z \in C}\{\Psi(x-z)\} .
$$

In practice, it is often highly desirable to have good upper bounds for the areas $\mathscr{H}^{n-1}\left(\rho^{-1}\{r\}\right)$ of the level sets $\rho^{-1}\{r\}$. For typical surface energies, which are usually within a constant factor of surface area, such bounds translate immediately into vital energy estimates.

General isoperimetric and relative isoperimetric inequalities provide useful lower bounds to these areas, but finding good upper bounds can be much more difficult.

If we have some estimate of the form

$$
\mathscr{L}^{n}\left(\rho^{-1}(I)\right) \leq A<\infty,
$$

where $I \subset \mathbb{R}^{1} \cap\{x: x \geq 0\}$ is an interval, we can use Federer's Coarea Formula [Federer 1969, 3.2.11] to prove there exists a value of $r$ in $I$ for which

$$
\mathscr{H}^{n-1}\left(\rho^{-1}\{r\}\right) \leq \frac{A}{\mathscr{L}^{1}(I)} .
$$

While these estimates are extremely useful in general, they do not provide good control over the value of $r$, and these upper bounds become quite useless when $\mathscr{L}^{1}(I)$ is too small. In particular, such an approach cannot be used to give an upper bound for $\mathscr{H}^{n-1}\left(\rho^{-1}\{r\}\right)$ for a specific value of $r$. 
Also, it may be that our goal is to estimate from above the volume of a region. Good upper bounds for $\mathscr{H}^{n-1}\left(\rho^{-1}\{r\}\right)$ for $\mathscr{L}^{1}$ every $r$, together with the Coarea Formula, which expresses the volume as the integral of these areas, would be extremely useful in such a situation. Such situations arise in both static and dynamical systems.

For a wide variety of flows (curvature flow, grain growth, surface diffusion, and so on), variational formulations involve energy terms that depend on the distance an interface has moved. Using the Coarea Formula, we can express the volume swept out by a moving interface over a given time interval as the integral of $\mathscr{H}^{n-1}\left(\rho^{-1}\{r\}\right)$, for an appropriate range of $r$.

For example, Almgren, Taylor, and Wang, in the very important paper [Almgren et al. 1993], consider general curvature flows of $n$-dimensional crystals $K(t)$. Their main results concern the existence, Hölder continuity, and regularity of such flows. Their Hölder continuity inequality is of the form

$$
\mathscr{L}^{n}(K(s) \triangle K(t)) \leq c|t-s|^{\alpha},
$$

where $\triangle$ as usual denotes symmetric difference. Their key estimate [Almgren et al. 1993, 4.2] is an upper bound for the areas $\mathscr{H}^{n-1}\left(\rho^{-1}\{r\}\right)$ in the special case where $\rho$ is ordinary Euclidean distance - i.e., where

$$
\Psi=\Psi_{E}: x \mapsto|x|
$$

is the Euclidean norm on $\mathbb{R}^{n}$. They use those upper bounds to obtain a good estimate of the form (1-1).

Our main result extends their estimate to more general distance functions, those induced by asymmetric norms that are $C^{2}$-smooth except at the origin. In the special case $\Psi=\Psi_{E}$, our estimate is sharper, exhibiting a better constant than the one in [Almgren et al. 1993].

Almgren and Wang [2000] gave an important extension of the model from [Almgren et al. 1993] by incorporating Gibbs-Thomson curvature effects. Here, too, estimates of the areas of level sets of distance functions play a central role.

Our results should prove useful for establishing Hölder continuity estimates for a wide variety of flows. They can also be used to estimate the speed with which an interface is moving.

The cases where $\mathscr{H}^{n-1}(C)=\infty$ (Remark 8) and where $n=1$ (Remark 9) are fairly trivial, so hereafter we assume that $\mathscr{H}^{n-1}(C)<\infty$ and $n \geq 2$.

By itself, the constraint $\mathscr{H}^{n-1}(C)<\infty$ does not do too much to preclude wild level sets, especially in the absence of any regularity assumptions on $C$ itself. Even if $C$ is smooth, the level sets $\rho^{-1}\{r\}$ might fail to be smooth altogether (if $\Psi$ is not smooth enough) or might be smooth only for small values of $r$ (for example, if $C$ consists of two disjoint spheres in $\mathbb{R}^{n}$ and $\Psi=\Psi_{E}$ ). 
Indeed, even if $C$ is the unit sphere in $\mathbb{R}^{n}$, in general there is no hope of finding useful a priori upper bounds for the areas $\mathscr{H}^{n-1}\left(\rho^{-1}\{r\}\right)$, for $\mathscr{L}^{1}$-almost every $r>0$, if $\Psi$ is an arbitrary function.

Because the Euclidean norm $\Psi_{E}$ is $C^{2}$-smooth on $\mathbb{R}^{n} \backslash\{\mathbf{0}\}$, it is natural for us to require that $\Psi$ be $C^{2}$-smooth on $\mathbb{R}^{n} \backslash\{\boldsymbol{0}\}$ in general.

We make no such smoothness assumptions on asymmetric norms until Section 4, where we need a reasonable upper bound for the distribution Laplacian of $\rho$. The assumption that $\Psi$ is $C^{2}$-smooth away from the origin is sufficient.

If $C$ were $C^{2}$-smooth, and if we had a priori upper bounds on its curvature, we would certainly expect to be able to find upper bounds for the areas $\mathscr{H}^{n-1}\left(\rho^{-1}\{r\}\right)$. In fact, even without the curvature bounds, the smoothness of $C$ alone would suffice.

In this paper, we do not assume that $C$ is even Lipschitz continuous. Instead, we make an extremely weak regularity assumption: that $C$ has uniform lower $(n-1)$ dimensional density ratio bounds at each point. This assumption does imply that $\mathscr{H}^{n-1}(C)>0$, which, when combined with the assumption that $\mathscr{H}^{n-1}(C)<\infty$, shows that $C$ must have Hausdorff dimension $n-1$. In particular, our main result does not hold if the Hausdorff dimension of $C$ is strictly less than $n-1$.

Our main theorem (Section 4) shows that, under the stated hypotheses on $\Psi$ and $C$, the areas $\mathscr{H}^{n-1}\left(\rho^{-1}\{r\}\right)$ can be very effectively bounded in terms of $\mathscr{H}^{n-1}(C)$ and $r^{n-1}$, and so the level sets do not grow too quickly.

For more general applicability, we state our results in terms of $\mathscr{H}^{n-1}\left(\rho^{-1}\{r\}\right)$ rather than in terms of $\mathscr{H}^{n-1}\left(\rho^{-1}\{r(t)\}\right)$. In fact, none of our results depends on the form of the function $r=r(t)$.

Because we rely on local estimates, our results and arguments are also independent of the diameter of the set $C$.

Our strategy is to use a general divergence theorem for sets of finite perimeter, along with the bounds on the distribution Laplacian of $\rho$, to establish estimates on the areas of level sets inside small balls (Sections 4.1 and 4.2). Then we combine those estimates using a covering argument, and we apply the lower density ratio bounds for $C$ to complete the proof of the main theorem (Section 4.3).

\section{Asymmetric norms}

Definition 1. An asymmetric norm on $\mathbb{R}^{n}$ is a function $\Psi: \mathbb{R}^{n} \rightarrow[0, \infty)$ which satisfies

(a) $\Psi(x) \geq 0$ for all $x \in \mathbb{R}^{n}$, with $\Psi(x)=0$ if and only if $x=\mathbf{0}$;

(b) $\Psi(c x)=c \Psi(x)$ whenever $c \geq 0$ and $x \in \mathbb{R}^{n}$;

(c) $\Psi(x+y) \leq \Psi(x)+\Psi(y)$ for all $x, y \in \mathbb{R}^{n}$ (triangle inequality).

If $p \in \mathbb{R}^{n}$ and $r \geq 0$, we let

$$
B^{n}(p, r)=\left\{x \in \mathbb{R}^{n}:|x-p| \leq r\right\}
$$


denote the closed (Euclidean) ball in $\mathbb{R}^{n}$ with center $p$ and radius $r$. More generally, if $\Psi$ is an asymmetric norm on $\mathbb{R}^{n}$, for each $r>0$ we let

$$
B_{\Psi}(p, r)=\left\{x \in \mathbb{R}^{n}: \Psi(x-p) \leq r\right\}
$$

denote the closed $\Psi$-ball in $\mathbb{R}^{n}$ with center $p$ and radius $r$. For brevity, we set

$$
B_{\Psi}(r)=B_{\Psi}(\mathbf{0}, r) .
$$

Because $\Psi$ is positively homogeneous of degree 1 , it suffices to study the unit balls $B_{\Psi}(1)$.

Here are some essential properties of asymmetric norms and their associated unit balls.

Theorem 2. Suppose $\Psi$ is an asymmetric norm on $\mathbb{R}^{n}$.

(1) $\Psi$ is a convex function on $\mathbb{R}^{n}$.

(2) $\Psi$ is continuous on $\mathbb{R}^{n}$. Define

$$
m=\inf \{|\Psi(v)|:|v|=1\}, \quad M=\sup \{|\Psi(v)|:|v|=1\} .
$$

Then $0<m \leq M<\infty$.

(3) $\Psi$ is Lipschitz continuous on $\mathbb{R}^{n}$, with constant $\operatorname{Lip} \Psi=M$.

(4) $\Psi$ is a norm if and only if it is an even function (meaning that $\Psi(-x)=\Psi(x)$ for all $x$ ).

(5) For each $r>0$, the ball $B_{\Psi}(r)$ is a compact, convex set containing the origin.

(6) There exist positive constants $c_{1}$ and $c_{2}$ for which

$$
c_{1}|x| \leq \Psi(x) \leq c_{2}|x| \quad \text { for all } x \in \mathbb{R}^{n} .
$$

The best such constants are $c_{1}=m$ and $c_{2}=M$.

(7) For all $x \in \mathbb{R}^{n}$,

$$
\Psi(x)=\frac{1}{z}, \quad \text { where } z=\sup \left\{\lambda: \lambda>0 \text { and } \lambda x \in B_{\Psi}(1)\right\} .
$$

Proof. Suppose $\lambda_{1}$ and $\lambda_{2}$ are nonnegative real numbers for which $\lambda_{1}+\lambda_{2}=1$. Suppose $x, y \in \mathbb{R}^{n}$. Then

$$
\Psi\left(\lambda_{1} x+\lambda_{2} y\right) \leq \Psi\left(\lambda_{1} x\right)+\Psi\left(\lambda_{2} y\right)=\lambda_{1} \Psi(x)+\lambda_{2} \Psi(y),
$$

which proves (1).

Since any convex function defined on a nonempty, open, convex subset of $\mathbb{R}^{n}$ is continuous on that set, it follows that $\Psi$ is continuous on $\mathbb{R}^{n}$. Since $\Psi$ is continuous, and since the unit ball in $\mathbb{R}^{n}$ is compact, the values $m$ and $M$ are actually attained for unit vectors $v$. Since $0<\Psi(v)<\infty$ whenever $v$ is not the zero vector, (2) follows.

We do not need the positive homogeneity of $\Psi$ to show that $\Psi$ is locally Lipschitz continuous, but we will use it for the global estimate. Fix any $x, y \in \mathbb{R}^{n}$. If $x=y$, clearly $|\Psi(x)-\Psi(y)| \leq M|x-y|$, as desired. 
Next we suppose that $x \neq y$. The relations $x=(x-y)+y$ and $y=(y-x)+x$ and the triangle inequality imply that

$$
\Psi(x)-\Psi(y) \leq \Psi(x-y), \quad \Psi(y)-\Psi(x) \leq \Psi(y-x),
$$

and therefore

$$
\begin{aligned}
|\Psi(x)-\Psi(y)| & \leq \max \{|\Psi(x-y)|,|\Psi(y-x)|\} \\
& =|x-y| \max \left\{\left|\Psi\left(\frac{x-y}{|x-y|}\right)\right|,\left|\Psi\left(\frac{y-x}{|y-x|}\right)\right|\right\} \leq M|x-y|,
\end{aligned}
$$

which completes the proof of (3).

If $\Psi$ is also even, then

$$
\Psi(c x)=c \Psi(x)=|c| \Psi(x)
$$

if $c \geq 0$, and

$$
\Psi(c x)=\Psi(-c x)=-c \Psi(x)=|c| \Psi(x)
$$

if $c<0$, so $\Psi$ is a norm. That completes the proof of (4).

Because $\Psi$ is positively homogeneous of degree 1, it suffices to prove (5) for the unit ball $B_{\Psi}(1)$. The convexity of $B_{\Psi}(1)$ follows immediately from the convexity of $\Psi$, and it is also clear that $B_{\Psi}(1)$ must be closed. We will show it is also bounded by comparing it to a multiple of the Euclidean norm.

Define $\Psi_{1}(x)=m|x|$ for all $x \in \mathbb{R}^{n}$. Clearly,

$$
B_{\Psi_{1}}(1)=\left\{x \in \mathbb{R}^{n}: m|x| \leq 1\right\}=B^{n}\left(\mathbf{0}, \frac{1}{m}\right) .
$$

Also, $\Psi(\mathbf{0})=\Psi_{1}(\mathbf{0})=0$, and, if $x \neq \mathbf{0}$,

$$
\Psi(x)=|x| \Psi\left(\frac{x}{|x|}\right) \geq m|x|=\Psi_{1}(x),
$$

which implies that $B_{\Psi}(1) \subset B_{\Psi_{1}}(1)=B^{n}(\mathbf{0}, 1 / m)$, and so $B_{\Psi}(1)$ is bounded, as needed.

We also define $\Psi_{2}(x)=M|x|$ for all $x \in \mathbb{R}^{n}$. Arguing as above, we see right away that $\Psi(x) \leq \Psi_{2}(x)$ for all $x$, and $B^{n}(\mathbf{0}, 1 / M)=B_{\Psi_{2}}(1) \subset B_{\Psi}(1)$. Therefore (6) holds with $c_{1}=m$ and $c_{2}=M$.

To see that $m$ and $M$ must be optimal, note that the inequality in (6) implies that

$$
c_{2} \geq \sup \left\{\frac{\Psi(x)}{|x|}: x \neq \mathbf{0}\right\}=\sup \left\{\Psi\left(\frac{x}{|x|}\right): x \neq \mathbf{0}\right\}=M,
$$

and likewise that $c_{1} \leq m$.

Finally, $z=\sup \left\{\lambda: \lambda>0\right.$ and $\left.\lambda x \in B_{\Psi}(1)\right\}=\sup \{\lambda: \lambda>0$ and $\Psi(\lambda x) \leq 1\}=$ $\sup \{\lambda: \lambda>0$ and $\lambda \Psi(x) \leq 1\}$. If $x \neq \mathbf{0}, \Psi(x)>0$, and clearly the supremum is obtained when $\lambda=1 / \Psi(x)$, which gives $z=1 / \Psi(x)$, as claimed in (7). If $x=\mathbf{0}$, we see that $z=\infty$, which correctly gives $\Psi(\mathbf{0})=0$ if we follow the convention that $1 / \infty=0$. 
Asymmetric norms have a wide variety of applications. Of course, all norms are asymmetric norms. Asymmetric norms which are not norms are also very important.

In many instances, a deviation (of, for example, the path of an object) in one direction may be more significant than a deviation in another direction.

While a typical norm would treat an overestimate and an equal underestimate the same way, an asymmetric norm easily allows these to be treated differently. In particular, it is easy to use asymmetric norms to derive alternative least-squares algorithms that use asymmetry to great advantage.

\section{Distance functions induced by asymmetric norms}

Associated with $\Psi$, we have distance functions $d_{p}$ to points $p \in \mathbb{R}^{n}$ :

$$
d_{p}(x)=\Psi(x-p)
$$

for all $x \in \mathbb{R}^{n}$. If $c_{1}$ and $c_{2}$ are any positive real numbers for which the inequality in Theorem 2(6) holds, then clearly

$$
c_{1}|x-p| \leq d_{p}(x) \leq c_{2}|x-p|
$$

for all $x, p \in \mathbb{R}^{n}$.

If $X$ is any nonempty subset of $\mathbb{R}^{n}$, we define the distance function to $X$ (induced by $\Psi)$ as follows:

$$
\operatorname{dist}_{\Psi}(x, X)=\inf \left\{d_{p}(x): p \in X\right\} .
$$

If $\Psi$ is even, $\operatorname{dist}_{\Psi}(\cdot, X)$ is the usual norm-induced distance function to $X$. In particular, when $\Psi(x)=|x|$, the function $\operatorname{dist}_{\Psi}(\cdot, X)=\operatorname{dist}(\cdot, X)$ is the ordinary Euclidean distance to the set $X$.

Theorem 3. Suppose $\Psi$ is an asymmetric norm on $\mathbb{R}^{n}$ and $X \subset \mathbb{R}^{n}$ is nonempty. Suppose $c_{1}$ and $c_{2}$ are any positive constants satisfying the inequality in Theorem 2(6). Then, for all $x \in \mathbb{R}^{n}$,

$$
c_{1} \operatorname{dist}(x, X) \leq \operatorname{dist}_{\Psi}(x, X) \leq c_{2} \operatorname{dist}(x, X) .
$$

Proof. Fix $x$ and pick any $\varepsilon>0$. Choose a point $p$ in $X$ satisfying

$$
|x-p| \leq \operatorname{dist}(x, X)+\frac{\varepsilon}{c_{2}} \text {. }
$$

Then

$$
\operatorname{dist}_{\Psi}(x, X) \leq \Psi(x-p) \leq c_{2}|x-p| \leq c_{2} \operatorname{dist}(x, X)+\varepsilon .
$$

Next we fix a point $p$ in $X$ for which

$$
\Psi(x-p) \leq \operatorname{dist}_{\Psi}(x, X)+\varepsilon .
$$

It follows that

$$
\operatorname{dist}_{\Psi}(x, X) \geq \Psi(x-p)-\varepsilon \geq c_{1}|x-p|-\varepsilon \geq c_{1} \operatorname{dist}(x, X)-\varepsilon,
$$


The result now follows since $\varepsilon$ may be arbitrarily small.

It is well-known that distance functions induced by norms are Lipschitz continuous. We show this is also the case for asymmetric norms:

Theorem 4 (The Lipschitz constant of dist $\Psi$ ). Suppose that $\Psi$ is an asymmetric norm on $\mathbb{R}^{n}$, and $m$ and $M$ are the constants given in Theorem 2. If $X$ is any nonempty subset of $\mathbb{R}^{n}$, the function

$$
\sigma(x)=\operatorname{dist}_{\Psi}(x, X)
$$

is Lipschitz continuous, with $\operatorname{Lip} \sigma=M$.

Proof. Suppose $x, y \in \mathbb{R}^{n}$. Since

$$
\sigma(x)=\inf \{\Psi(x-p): p \in X\},
$$

for any $p \in X$ we have $\sigma(x) \leq \Psi(x-p)$. Also, for any $\varepsilon>0$, there exists a point $p \in X$ such that

$$
\sigma(x) \leq \Psi(x-p) \leq \sigma(x)+\varepsilon|x-y| .
$$

Then

$$
\sigma(y) \leq \Psi(y-p) \leq \Psi(y-x)+\Psi(x-p) \leq \sigma(x)+(M+\varepsilon)|x-y|,
$$

which gives

$$
\sigma(y)-\sigma(x) \leq M|x-y|
$$

once we let $\varepsilon \rightarrow 0$. The same argument with $x$ and $y$ switched gives

$$
\sigma(x)-\sigma(y) \leq M|x-y| .
$$

Theorem 5. Suppose $X \subset \mathbb{R}^{n}$ is nonempty and compact, $\Psi$ is an asymmetric norm on $\mathbb{R}^{n}$, and $\sigma(x)=\operatorname{dist}_{\Psi}(x, X)$ is the $\Psi$-induced distance to the set $X$. Suppose $m$ and $M$ are the constants given in Theorem 2. Then, for $\mathscr{L}^{n}$-almost every $x \in \mathbb{R}^{n} \backslash X$, the function $\sigma$ is differentiable and

$$
m \leq|(\nabla \sigma)(x)| \leq M .
$$

Proof. Since $\sigma$ is Lipschitz continuous, Rademacher's theorem implies that $\sigma$ is differentiable for $\mathscr{L}^{n}$-almost every $x$. Fix any such point $x \in \mathbb{R}^{n} \backslash X$. Then $(\nabla \sigma)(x)$ exists, as does the directional derivative

$$
\left(D_{v} \sigma\right)(x)=(\nabla \sigma)(x) \cdot v
$$

for any unit vector $v \in \mathbb{R}^{n}$.

Since $X$ is closed, there exists a point $z$, which depends on $x$, for which $\sigma(x)=$ $\Psi(x-z)$. Fix $z$ and let $y=x+t v$ for $t>0$ and for any unit vector $v$. Then

$$
\sigma(y) \leq \Psi(y-z) \leq \Psi(y-x)+\Psi(x-z)=\Psi(y-x)+\sigma(x),
$$

and so

$$
\frac{\sigma(x+t v)-\sigma(x)}{t}=\frac{\sigma(y)-\sigma(x)}{|y-x|} \leq \Psi\left(\frac{y-x}{|y-x|}\right) \leq M .
$$


We set $v=\frac{(\nabla \sigma)(x)}{|(\nabla \sigma)(x)|}$ and let $t \rightarrow 0$ to deduce that $|(\nabla \sigma)(x)| \leq M$.

To prove the lower bound for $|(\nabla \sigma)(x)|$, we let

$$
y=(1-t) x+t z \text { for } 0 \leq t \leq 1,
$$

so that

$$
\Psi(x-z)=\Psi(x-y)+\Psi(y-z) .
$$

It follows that

$$
\begin{aligned}
\sigma(y) & \leq \Psi(y-z)=\Psi(x-y)+\Psi(y-z)-\Psi(x-y) \\
& =\Psi(x-z)-\Psi(x-y)=\sigma(x)-\Psi(x-y),
\end{aligned}
$$

and so

$$
\frac{\sigma(y)-\sigma(x)}{|y-x|} \leq-\Psi\left(\frac{y-x}{|y-x|}\right) \leq-m .
$$

Letting $t \rightarrow 0$ gives $-|(\nabla \sigma)(x)| \leq\left(D_{v} \sigma\right)(x) \leq-m$, and the result follows.

\section{The main result}

Theorem 6. Suppose $n \geq 2$. Suppose $C$ is a nonempty compact subset of $\mathbb{R}^{n}$ with $\mathscr{H}^{n-1}(C)<\infty, \Psi$ is an asymmetric norm on $\mathbb{R}^{n}$,

$$
\rho(x)=\operatorname{dist}_{\Psi}(x, C)
$$

for each $x \in \mathbb{R}^{n}$, and the restriction of $\Psi$ to $\mathbb{R}^{n} \backslash\{\mathbf{0}\}$ is $C^{2}$-smooth:

$$
\Psi \mid \mathbb{R}^{n} \backslash\{\mathbf{0}\} \in C^{2} .
$$

Suppose also that there exist positive constants $\delta$ and $\theta$ such that

$$
\frac{\mathscr{H}^{n-1}\left(C \cap B^{n}(p, \gamma)\right)}{\gamma^{n-1}} \geq \theta
$$

for all $p \in C$ and $\gamma \in(0, \delta]$. Then, for $\mathscr{L}^{1}$-almost every $r \in(0, \infty)$,

$$
\mathscr{H}^{n-1}\left(\rho^{-1}\{r\}\right) \leq\left(\frac{\mu_{n} \beta(n) m^{n-1}}{\theta}\right) \sup \left\{1,\left(\frac{r}{m \delta}\right)^{n-1}\right\} \mathscr{H}^{n-1}(C),
$$

where

$$
\begin{aligned}
0<m & =\inf \left\{\frac{\Psi(x)}{|x|}: x \neq \mathbf{0}\right\} \leq \sup \left\{\frac{\Psi(x)}{|x|}: x \neq \mathbf{0}\right\}=M<\infty, \\
C_{L} & =\sup \{(\Delta \Psi)(x):|x|=1\}, \\
\mu_{n} & =\frac{M}{m} \frac{\alpha(n) 4^{n}\left(1+\sqrt{C_{L}}\right)^{2}}{m^{n}},
\end{aligned}
$$

$\alpha(n)$ is the volume of the unit ball in $\mathbb{R}^{n}$, and $\beta(n)$ is the optimal constant from the Besicovitch Covering Theorem. 
Remark 7. In the special case where $\Psi(x)=|x|$, our estimate is a little stronger than the original estimate from [Almgren et al. 1993, 4.2], which was proven using a similar strategy. In this special case, clearly $m=M=1$, and $C_{L}=n-1$.

Remark 8. If $\mathscr{H}^{n-1}(C)=\infty$, obviously (4-1) is true as well. Since we assume (without loss of generality) that $\mathscr{H}^{n-1}(C)<\infty$, the uniform lower density ratio bounds imply that $\mathcal{H}^{n-1}(C)>0$, and so $C$ has Hausdorff dimension $n-1$.

Remark 9. Since $\mathscr{H}^{0}$ is just counting measure, it is easy to derive a corresponding (but not very interesting) result for the case $n=1$ :

$$
\mathscr{H}^{0}\left(\rho^{-1}\{r\}\right) \leq 2 \mathscr{H}^{0}(C)
$$

for all $r>0$. This result holds for arbitrary nonempty compact subsets $C \subset \mathbb{R}^{1}$ and for arbitrary asymmetric norms $\Psi$. If $\mathscr{H}^{0}(C)=\infty$, the result is immediate. If $C=\left\{p_{1}, p_{2}, \ldots, p_{N}\right\}$, where $N<\infty$, we note that, for each $r>0$,

$\mathscr{H}^{0}\left(\rho^{-1}\{r\}\right) \leq \mathscr{H}^{0}\left(\bigcup_{i=1}^{N}\left\{x \in \mathbb{R}^{1}: \Psi\left(x-p_{i}\right)=r\right\}\right) \leq \sum_{i=1}^{N} \mathscr{H}^{0}\left(\left\{x \in \mathbb{R}^{1}: \Psi\left(x-p_{i}\right)=r\right\}\right)$,

and the result follows.

Our proof of Theorem 6 proceeds in several steps, given in the next three sections. Hereafter, we assume that $C, \Psi, m, M, C_{L}, \alpha(n), \beta(n)$, and $\mu_{n}$ are as stated in the theorem.

4.1. A divergence theorem estimate. The following theorem shows, essentially, that the $(n-1)$-dimensional area of the level sets does not grow too quickly compared to the $n$-dimensional volume swept out.

Our proof proceeds by finding lower and upper bounds to the integral of the Laplacian of a regularization $\sigma_{\varepsilon}$ of $\sigma$, and then letting $\varepsilon$ go to zero.

The upper bound holds because of the bound $C_{L}$ on the Laplacian of $\Psi$. This estimate leads to an upper bound on the Laplacian of the $\sigma$-induced distance functions to a point, and then to an upper bound on the distribution Laplacian of the infimum $\sigma$ of a countable collection of such functions.

The lower bound is an application of the divergence theorem for sets of finite perimeter (noting that $\nabla \sigma$ is normal to the level sets of $\sigma$ ); the key estimates are $a$ priori lower and upper bounds for $|\nabla \sigma|$ on level sets; see Theorem 5.

Good references for sets of finite perimeter include [Ambrosio et al. 2000; Evans and Gariepy 1992; Federer 1969; Krantz and Parks 1999; Mattila 1995].

Below, $n_{\Omega}(x)$ is the measure-theoretic exterior unit normal to $\Omega$ at $x$, as defined for sets of finite perimeter. (See [Federer 1969, § 4.5.5], for example.]

Remark 10. Theorem 11 and Proposition 13 will not be applied directly to the entire set $C$. To avoid confusion, we will use $X$ and $\sigma$ in place of $C$ and $\rho$ in the statements of these results. 
Theorem 11. Suppose $n \geq 2$. Let $X$ be a nonempty compact subset of $\mathbb{R}^{n}$. Suppose $\Psi, m, M$, and $C_{L}$ are as stated in Theorem 6 , and suppose that $\sigma(x)=\operatorname{dist}_{\Psi}(x, X)$ for all $x \in \mathbb{R}^{n}$.

Then, for $\mathscr{L}^{1}$-almost every $R \in(0, \infty)$ and for $\mathscr{L}^{1}$-almost every $S \in(R, \infty)$,

$$
\Omega=\sigma^{-1}(R, S)=\left\{x \in \mathbb{R}^{n}: R<\sigma(x)<S\right\}
$$

is a set of finite perimeter with the following properties:

(1) $\mathscr{H}^{n-1}(\partial \Omega)<\infty$, and

$$
\mathscr{H}^{n-1}\left((\partial \Omega) \triangle\left(\sigma^{-1}\{R\} \cup \sigma^{-1}\{S\}\right)\right)=0,
$$

where $A \triangle B$ is the symmetric difference of $A$ and $B$.

(2) For $\mathscr{H}^{n-1}$-almost every $x \in \sigma^{-1}\{R\}$, the function $\sigma$ is differentiable,

$$
m \leq|(\nabla \sigma)(x)| \leq M,
$$

and

$$
n_{\Omega}(x)=-\frac{(\nabla \sigma)(x)}{|(\nabla \sigma)(x)|} .
$$

The same holds true (with the sign of $n_{\Omega}(x)$ reversed) for $\mathscr{H}^{n-1}$-almost every $x \in \sigma^{-1}\{S\}$.

$$
m \mathscr{H}^{n-1}\left(\sigma^{-1}\{S\}\right)-M \mathscr{H}^{n-1}\left(\sigma^{-1}\{R\}\right) \leq \frac{M C_{L}}{R} \mathscr{L}^{n}(\Omega) .
$$

Proof. Since $X$ is compact, for each $N>0$ we have

$$
\mathscr{L}^{n}\left(\sigma^{-1}(0, N)\right)<\infty .
$$

Conclusions (1) and (2) are then straightforward consequences of the Coarea Formula [Federer 1969, 3.2.11], together with Theorem 5 and the chain rule.

Suppose that $\eta: \mathbb{R}^{n} \rightarrow[0, \infty)$ is of class $C^{\infty}$, with suppt $\eta \subset B^{n}(\mathbf{0}, 1)$ and $\int_{\mathbb{R}^{n}} \eta d \mathscr{L}^{n}=1$. Define the smooth mollifier $\eta_{\varepsilon}$ in the usual way:

$$
\eta_{\varepsilon}(x)=\frac{1}{\varepsilon^{n}} \eta\left(\frac{x}{\varepsilon}\right) .
$$

Then $\eta_{\varepsilon}$ is also of class $C^{\infty}$ and is supported in $B^{n}(\mathbf{0}, \varepsilon)$.

Let $\sigma_{\varepsilon}$ be the convolution of $\sigma$ with $\eta_{\varepsilon}$ :

$$
\sigma_{\varepsilon}=\sigma * \eta_{\varepsilon} .
$$

Then $\sigma_{\varepsilon} \rightarrow \sigma$ uniformly and $\nabla \sigma_{\varepsilon} \rightarrow \nabla \sigma$ pointwise almost everywhere as $\varepsilon \rightarrow 0$. Suppose $R$ and $S$ satisfy properties (1) and (2). Let $\Omega=\sigma^{-1}(R, S)$. For $\mathscr{L}^{1}$-almost every such $R \in(0, \infty)$ and for $\mathscr{L}^{1}$-almost every such $S \in(R, \infty)$, we have

$$
\lim _{\varepsilon \rightarrow 0} \int_{\partial \Omega}\left|\nabla \sigma-\nabla \sigma_{\varepsilon}\right| d \mathscr{H}^{n-1}=0 .
$$


For the remainder of the proof, we will assume that $R$ and $S$ satisfy properties (1) and (2) above and Equation (4-2). The proof of (3) proceeds by showing that (4-3) $m \mathscr{H}^{n-1}\left(\sigma^{-1}\{S\}\right)-M \mathscr{H}^{n-1}\left(\sigma^{-1}\{R\}\right) \leq \lim _{\varepsilon \rightarrow 0} \int_{\Omega} \Delta \sigma_{\varepsilon} d \mathscr{L}^{n} \leq \frac{M C_{L}}{R} \mathscr{L}^{n}(\Omega)$.

Let $\chi_{\Omega}$ be the characteristic function of $\Omega$. Then, for any $\varepsilon>0$,

$$
\begin{aligned}
\int_{\Omega} \Delta \sigma_{\varepsilon} d \mathscr{L}^{n} & =\lim _{\delta \rightarrow 0} \int_{\mathbb{R}^{n}}\left(\chi_{\Omega} * \eta_{\delta}\right) \Delta \sigma_{\varepsilon} d \mathscr{L}^{n} \\
& =\lim _{\delta \rightarrow 0} \int_{\mathbb{R}^{n}}\left(\Delta\left(\chi_{\Omega} * \eta_{\delta}\right)\right) \sigma_{\varepsilon} d \mathscr{L}^{n} \\
& =\lim _{\delta \rightarrow 0} \int_{\mathbb{R}^{n}}\left(\Delta\left(\chi_{\Omega} * \eta_{\delta} * \eta_{\varepsilon}\right)\right) \sigma d \mathscr{L}^{n} .
\end{aligned}
$$

The first equality above holds because $\chi_{\Omega} * \eta_{\delta} \rightarrow \chi_{\Omega}$ uniformly as $\delta \rightarrow 0$. The second follows from the integration by parts formula. The third equality holds with $\Delta$ replaced by $D_{i i}$ for each $i=1,2,3, \ldots, n$, and so the result follows.

Fix $\delta>0$. Outside the set $\Omega^{\prime}=\{x: \operatorname{dist}(x, \Omega) \leq \varepsilon+\delta\}$, the integrand in (4-4) vanishes. Suppose $x \in \Omega^{\prime}$ and $p \in X$.

Claim 12. $\left(\Delta d_{p}\right)(x) \leq \frac{C_{L}}{R / M-\delta-\varepsilon} ;$ moreover, for all nonnegative test functions $\varphi$ supported in $\Omega^{\prime}$,

$$
\int_{\Omega^{\prime}}(\Delta \varphi) d_{p} d \mathscr{L}^{n} \leq \frac{C_{L}}{R / M-\delta-\varepsilon} \int_{\Omega^{\prime}} \varphi d \mathscr{L}^{n} .
$$

Proof. The upper bound on the Laplacian of $\Psi$ (on unit vectors) gives (after translating and scaling appropriately)

$$
\left(\Delta d_{p}\right)(x) \leq \frac{C_{L}}{|x-p|} .
$$

The first inequality in the claim is then a consequence of the following lower bound on $|x-p|$ : $|x-p| \geq \inf \{\operatorname{dist}(x, X): x \in \Omega\}-\delta-\varepsilon \geq \inf \left\{\frac{\sigma(x)}{M}: x \in \Omega\right\}-\delta-\varepsilon \geq \frac{R}{M}-\delta-\varepsilon$. The second inequality follows from the first and the integration by parts formula.

The next step is to use this pointwise bound to obtain an upper bound on the distribution Laplacian of $\sigma$. In general (see [Almgren et al. 1993, 4.2]), if $f$ and $g$ are summable functions such that (for some $k>0$ )

$$
\sup \left\{\int_{\Omega^{\prime}}(\Delta \varphi) f d \mathscr{L}^{n}, \int_{\Omega^{\prime}}(\Delta \varphi) g d \mathscr{L}^{n}\right\} \leq k \int_{\Omega^{\prime}} \varphi d \mathscr{L}^{n}
$$


for each nonnegative test function $\varphi$ supported in $\Omega^{\prime}$, then

$$
\int_{\Omega^{\prime}}(\Delta \varphi) \inf \{f, g\} d \mathscr{L}^{n} \leq k \int_{\Omega^{\prime}} \varphi d \mathscr{L}^{n} .
$$

Note that $\sigma$ is the infimum of a countable set of functions $\left\{d_{p_{i}}\right\}$, where the $p_{i}$ 's are a countable, dense subset of $X$, and use Lebesgue's Dominated Convergence Theorem and Claim 12 to conclude that

$$
\int_{\Omega^{\prime}}(\Delta \varphi) \sigma d \mathscr{L}^{n} \leq \frac{C_{L}}{R / M-\delta-\varepsilon} \int_{\Omega^{\prime}} \varphi d \mathscr{L}^{n}
$$

for each $\varphi$ (as above). Using (4-4) and applying this result with $\varphi=\chi_{\Omega} * \eta_{\delta} * \eta_{\varepsilon}$ yields

$$
\begin{aligned}
\int_{\Omega} \Delta \sigma_{\varepsilon} d \mathscr{L}^{n} & =\lim _{\delta \rightarrow 0} \int_{\Omega^{\prime}}\left(\Delta\left(\chi_{\Omega} * \eta_{\delta} * \eta_{\varepsilon}\right)\right) \sigma d \mathscr{L}^{n} \\
& \leq \lim _{\delta \rightarrow 0} \frac{C_{L}}{R / M-\delta-\varepsilon} \int_{\Omega^{\prime}}\left(\chi_{\Omega} * \eta_{\delta} * \eta_{\varepsilon}\right) d \mathscr{L}^{n} \\
& =\frac{C_{L}}{R / M-\varepsilon} \int_{\Omega^{\prime}}\left(\chi_{\Omega} * \eta_{\varepsilon}\right) d \mathscr{L}^{n} .
\end{aligned}
$$

Letting $\varepsilon \rightarrow 0$ completes the proof of the second inequality in (4-3).

The idea behind proving the first inequality in (4-3) is to apply the Divergence Theorem and (1) and (2) in $\Omega$ :

$$
\begin{aligned}
& \int_{\Omega} \Delta \sigma_{\varepsilon} d \mathscr{L}^{n}=\int_{\Omega} \operatorname{div} \nabla \sigma_{\varepsilon} d \mathscr{L}^{n}=\int_{\partial \Omega} \nabla \sigma_{\varepsilon} \cdot n_{\Omega} d \mathscr{H}^{n-1} \\
& =\int_{\sigma^{-1}\{S\}}(\nabla \sigma) \cdot \frac{\nabla \sigma(x)}{|\nabla \sigma(x)|} d \mathscr{H}^{n-1}-\int_{\sigma^{-1}\{R\}}(\nabla \sigma) \cdot \frac{\nabla \sigma(x)}{|\nabla \sigma(x)|} d \mathscr{H}^{n-1} \\
& +\int_{\sigma^{-1}\{S\} \cup \sigma^{-1}\{R\}}\left(\nabla \sigma_{\varepsilon}-\nabla \sigma\right) \cdot n_{\Omega} d \mathscr{H}^{n-1} \\
& =\int_{\sigma^{-1}\{S\}}|\nabla \sigma(x)| d \mathscr{H}^{n-1}-\int_{\sigma^{-1}\{R\}}|\nabla \sigma(x)| d \mathscr{H}^{n-1} \\
& +\int_{\sigma^{-1}\{S\} \cup \sigma^{-1}\{R\}}\left(\nabla \sigma_{\varepsilon}-\nabla \sigma\right) \cdot n_{\Omega} d \mathscr{H}^{n-1} \\
& \geq m \mathscr{H}^{n-1}\left(\sigma^{-1}\{S\}\right)-M \mathscr{H}^{n-1}\left(\sigma^{-1}\{R\}\right) \\
& +\int_{\sigma^{-1}\{S\} \cup \sigma^{-1}\{R\}}\left(\nabla \sigma_{\varepsilon}-\nabla \sigma\right) \cdot n_{\Omega} d \mathscr{H}^{n-1} .
\end{aligned}
$$

Since (4-2) implies that

$$
\lim _{\varepsilon \rightarrow 0} \int_{\sigma^{-1}\{S\} \cup \sigma^{-1}\{R\}}\left(\nabla \sigma_{\varepsilon}-\nabla \sigma\right) \cdot n_{\Omega} d \mathscr{H}^{n-1}=0,
$$

the left-hand inequality in (4-3) follows by letting $\varepsilon \rightarrow 0$. Statement (3) is therefore proven. 


\subsection{The area of level sets of $\sigma$ inside balls.}

Proposition 13. Suppose $n \geq 2$. Let $X$ be a compact subset of $\mathbb{R}^{n}$. Suppose that $\Psi, m, M, C_{L}, \alpha(n), \beta(n)$, and $\mu_{n}$ are as stated in Theorem 6, and define $\sigma(x)=\operatorname{dist}_{\Psi}(x, X)$ for all $x \in \mathbb{R}^{n}$. Then, for any point $q \in X$ and for $\mathscr{L}^{1}$-almost every $r>0$, we have

$$
\mathscr{H}^{n-1}\left(\left(\sigma^{-1}\{r\}\right) \cap B^{n}\left(q, \frac{2}{m} r\right)\right) \leq \mu_{n} r^{n-1} .
$$

Proof. It suffices to consider the case $r=1$, for then the proposition follows by scaling by the factor $r$. Since only boundaries inside $B^{n}\left(q, \frac{2}{m}\right)$ are being considered, and since $r=1$, there is no loss of generality in assuming that $X \subset B^{n}\left(q, \frac{3}{m}\right)$. Taking $S=1$ in Theorem 11(3) then gives

$$
m \mathscr{H}^{n-1}\left(\sigma^{-1}\{1\}\right) \leq M \mathscr{H}^{n-1}\left(\sigma^{-1}\{R\}\right)+\frac{M C_{L}}{R} \mathscr{L}^{n}\left(\sigma^{-1}(R, 1)\right)
$$

for $\mathscr{L}^{1}$-almost every $R \in(0,1)$. Since $X \subset B^{n}(q, 3 / m)$, we have $\sigma^{-1}(R, 1) \subset$ $B^{n}(q, 4 / m)$, and so

$$
\mathscr{L}^{n}\left(\sigma^{-1}(R, 1)\right) \leq \alpha(n) \frac{4^{n}}{m^{n}} .
$$

For any $w \in(0,1)$, the Coarea Formula [Federer 1969, 3.2.11] guarantees the existence of $R \in(w, 1)$ for which

$$
\mathscr{H}^{n-1}\left(\sigma^{-1}\{R\}\right) \leq \alpha(n) \frac{4^{n}}{(1-w) m^{n}},
$$

and so (4-5), (4-6), and (4-7) imply that

$$
\begin{aligned}
\mathscr{H}^{n-1}\left(\sigma^{-1}\{1\}\right) & \leq \frac{M}{m} \alpha(n) \frac{4^{n}}{m^{n}} \frac{1}{1-w}+\frac{M}{m} \alpha(n) \frac{4^{n}}{m^{n}} \frac{C_{L}}{w} \\
& =\frac{M}{m} \alpha(n) \frac{4^{n}}{m^{n}}\left(\frac{1}{1-w}+\frac{C_{L}}{w}\right) .
\end{aligned}
$$

Letting

$$
h(w)=\frac{1}{1-w}+\frac{C_{L}}{w},
$$

elementary calculus shows that $h(w)$ achieves its minimum value in $(0,1)$,

$$
h\left(w_{\min }\right)=\left(1+\sqrt{C_{L}}\right)^{2},
$$

when

$$
w_{\min }=\frac{\sqrt{C_{L}}}{\sqrt{C_{L}}+1} .
$$

Substituting this into (4-8), we get

$$
\mathscr{H}^{n-1}\left(\sigma^{-1}\{1\}\right) \leq \frac{M}{m} \alpha(n) \frac{4^{n}}{m^{n}}\left(1+\sqrt{C_{L}}\right)^{2}=\mu_{n},
$$

as desired. 
4.3. The covering step. Suppose $n \geq 2$ and let $C$ be as stated in Theorem 6. Fix any $r \in(0, \infty)$, and let $\mathscr{F}^{*}$ be the collection of all closed balls $B^{n}\left(p, \frac{1}{m} r\right)$, centered at points $p \in C$. The Besicovitch Covering Theorem (see [Evans and Gariepy 1992, 1.5.2], for example) guarantees that there exist subfamilies $\mathscr{F}_{1}, \mathscr{F}_{2}, \ldots, \mathscr{F}_{\beta(n)}$ of

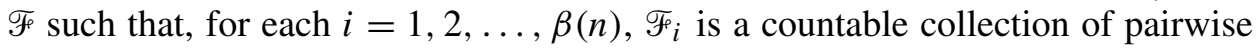
disjoint balls in $\mathscr{F}$, and

$$
C \subset \bigcup_{i=1}^{\beta(n)} \bigcup_{B \in \mathscr{F}_{i}} B
$$

Since $C$ is compact, we can take the subfamilies to be finite and thereby obtain a finite subcover. In other words, there exists a finite collection of points $p_{1}, p_{2}, \ldots, p_{N} \in C$ such that

$$
C \subset \bigcup_{i=1}^{N} B^{n}\left(p_{i}, \frac{1}{m} r\right)
$$

and no point is covered more than $\beta(n)$ times. Therefore,

$$
\sum_{i=1}^{N} \mathscr{H}^{n-1}\left(C \cap B^{n}\left(p_{i}, \frac{1}{m} r\right)\right) \leq \beta(n) \mathscr{H}^{n-1}(C) .
$$

Also,

$$
\rho^{-1}\{r\} \subset \bigcup_{i=1}^{N} B^{n}\left(p_{i}, \frac{2}{m} r\right),
$$

since each point $x$ in $\rho^{-1}\{r\}$ is no further than $r$ (in $\rho$-distance, hence $r / m$ in Euclidean distance) from some point $x^{\prime} \in C$, which in turn is at a Euclidean distance of no more than $r / m$ from one of the $p_{i}$. Proposition 13 and (4-10) imply

$$
\mathscr{H}^{n-1}\left(\rho^{-1}\{r\}\right) \leq \sum_{i=1}^{N} \mathscr{H}^{n-1}\left(\rho^{-1}\{r\} \cap B^{n}\left(p_{i}, \frac{2}{m} r\right)\right) \leq \sum_{i=1}^{N} \mu_{n} r^{n-1} .
$$

Next, the lower density ratio bounds in $C$ will be used to obtain an appropriate upper bound for $r^{n-1}$. If $r / m \leq \delta$ then, for each $i$,

$$
r^{n-1} \leq \frac{m^{n-1}}{\theta} \mathscr{H}^{n-1}\left(C \cap B^{n}\left(p_{i}, \frac{1}{m} r\right)\right) .
$$

Combining this result with (4-11) and (4-9), we get

$\mathscr{H}^{n-1}\left(\rho^{-1}\{r\}\right) \leq \frac{\mu_{n} m^{n-1}}{\theta} \sum_{i=1}^{N} \mathscr{H}^{n-1}\left(C \cap B^{n}\left(p_{i}, \frac{1}{m} r\right)\right) \leq \frac{\mu_{n} m^{n-1} \beta(n)}{\theta} \mathscr{H}^{n-1}(C)$,

which verifies (4-1) if $r / m \leq \delta$. 
If $r / m>\delta$, an application of the uniform lower density ratio bounds at radius $\delta$ yields

$$
\begin{aligned}
r^{n-1} & =\left(\frac{r}{m \delta}\right)^{n-1} m^{n-1}(\delta)^{n-1} \\
& \leq\left(\frac{r}{m \delta}\right)^{n-1} m^{n-1} \frac{\mathscr{H}^{n-1}\left(C \cap B^{n}\left(p_{i}, \delta\right)\right)}{\theta} \\
& \leq\left(\frac{r}{m \delta}\right)^{n-1} m^{n-1} \frac{\mathcal{H}^{n-1}\left(C \cap B^{n}\left(p_{i}, \frac{1}{m} r\right)\right)}{\theta} .
\end{aligned}
$$

It follows that

$$
\mathscr{H}^{n-1}\left(\rho^{-1}\{r\}\right) \leq\left(\frac{r}{m \delta}\right)^{n-1} \frac{\mu_{n} m^{n-1} \beta(n)}{\theta} \mathscr{H}^{n-1}(C) .
$$

This completes the proof of Theorem 6 .

\section{References}

[Almgren and Wang 2000] F. Almgren and L. Wang, "Mathematical existence of crystal growth with Gibbs-Thomson curvature effects", J. Geom. Anal. 10:1 (2000), 1-100. MR 2001f:49080

[Almgren et al. 1993] F. Almgren, J. E. Taylor, and L. Wang, "Curvature-driven flows: a variational approach”, SIAM J. Control Optim. 31:2 (1993), 387-438. MR 94h:58067 Zbl 0783.35002

[Ambrosio et al. 2000] L. Ambrosio, N. Fusco, and D. Pallara, Functions of bounded variation and free discontinuity problems, Oxford Mathematical Monographs, Oxford University Press, New York, 2000. MR 2003a:49002

[Evans and Gariepy 1992] L. C. Evans and R. F. Gariepy, Measure theory and fine properties of functions, Studies in Advanced Mathematics, CRC Press, Boca Raton, FL, 1992. MR 93f:28001

[Federer 1969] H. Federer, Geometric measure theory, Grundlehren der math. Wissenschaften 153, Springer, New York, 1969. MR 41 \#1976

[Krantz and Parks 1999] S. G. Krantz and H. R. Parks, The geometry of domains in space, Birkhäuser Advanced Texts: Basler Lehrbücher, Birkhäuser, Boston, 1999. MR 2000m:28005

[Mattila 1995] P. Mattila, Geometry of sets and measures in Euclidean spaces: Fractals and rectifiability, Cambridge Studies in Advanced Mathematics 44, Cambridge University Press, Cambridge, 1995. MR 96h:28006

Received August 24, 2002. Revised January 2, 2003.

DaVid G. CARABALlo

DEPARTMENT OF MATHEMATICS

REISS SCIENCE BUILDING, 2ND FLOOR

GEORGETOWN UNIVERSITY

WASHINGTON, DC 20057-1233

UNITED STATES

dgc3@georgetown.edu 